\title{
Haptic Display Using Fishing Rod
}

\author{
Daiki Naito $^{(\bowtie)}$ and Hiroyuki Kajimoto ${ }^{(\bowtie)}$ \\ The University of Electro-Communications, 1-5-1 Chofugaoka, \\ Chofu, Tokyo, Japan \\ \{naito,kajimoto\}@kaji-lab.jp
}

\begin{abstract}
This paper proposes a grounded haptic display that can widely present a force sensation using a fishing rod. The proposed haptic display incorporates a fishing rod to present the force sensation at the user's fingertip using a thread being reeled from the tip of the fishing rod. The multidirectional force sensation is presented by driving the base supporting the fishing rod using a pan-tilt mechanism, and wending the thread using the reel. The fishing rod is lightweight and moves at high speed. Additionally, it is possible to widely present the force by bending the fishing rod. This device requires conversion from the position of the user's fingertip and magnitude of the force to the necessary winding force of the thread and the posture of the fishing rod. This study developed a control method and evaluated it by considering a case wherein the force to be presented is exerted in the vertical direction.
\end{abstract}

Keywords: Virtual reality $\cdot$ Human interface $\cdot$ Haptic device $\cdot$ Fishing rod

\section{Introduction}

In the field of virtual reality, many studies are developing sensory presentation devices that reproduce sensations felt in the real world. Presenting a force sensation is an important element with regard to improving the perception of objects in virtual space [1].

A haptic display is a system that accurately tracks the movement of the user and presents the required force to their fingertips and tools [2]. Users can perceive the sensation of touching virtual objects created by a computer using the haptic display [35]. To present a force in an arbitrary direction and at an arbitrary point in space, the first basic structure to be considered is a structure using the robot arm of a serial link such as PHANToM [4]. One drawback of this type is that, to realize a large workspace, a rigid bar with the same length as the workspace moves within the space, which increases the risk and requires a motor with a large output at the base to generate a force at the end of the rigid bar. Another possible structure is the use of thread traction, as in the case of

Electronic supplementary material The online version of this chapter (https://doi.org/10.1007/ 978-3-030-58147-3_36) contains supplementary material, which is available to authorized users. 
SPIDAR [5]. This device type has a problem that numerous winding mechanisms must be installed to cover the entire workspace. Hence, a large-scale device is required to realize a wide workspace.

To this end, we propose a new type of haptic display that can present a force sensation to the fingertips using a fishing rod shown in Fig. 1. The force sensation is presented to the finger by attaching a thread to the finger and controlling the winding force of the thread. The base of the fishing rod has a pan-tilt mechanism. Because the fishing rod is lightweight, even a motor with a relatively low output can control its attitude at high speed. Although there is a restriction whereby the pulling direction of the thread cannot be set in a perfectly arbitrary manner (for example, a downward force cannot be generated), this might serve as a technique with a wide workspace, in the sense of range.

This paper presents the configuration of the proposed system and the development
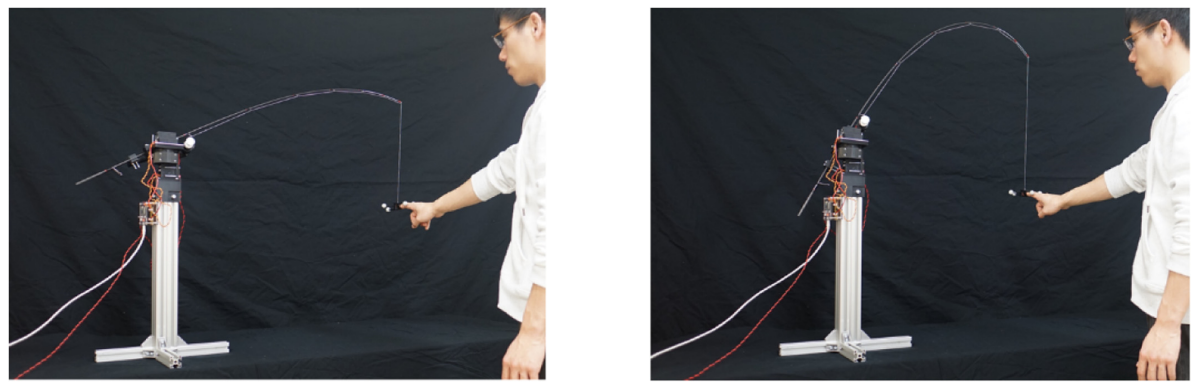

Fig. 1. Overview of device: (left) fishing rod is not bent; (right) fishing rod is bent.

and evaluation of a control method in the case wherein the force direction is vertical.

\section{Related Work}

Here, the haptic displays using a thread are described. The thread-based haptic displays can be approximately divided into wearable type and grounded type displays.

The wearable type uses a technique for presenting the force sensation by mounting the device on the user's body. Hirose developed HapticGEAR [6] and Hosseini developed a similar device using twisted-thread actuators [7]. In this wearable type of haptic displays, the range wherein the force sensation can be exerted is wide, and the user can use it freely. However, wearing the device is time-consuming and imposes a burden to the user owing to the weight of the device. Additionally, the reaction force is inevitably generated in the mounting part when the force sensation is presented.

In contrast, a grounded thread-based display type, such as SPIDAR [5], fixes the device to the wall or floor. The device essentially comprises a cube type frame and four motors at the vertices, and exerts the force sensation to the finger using winding threads. The grounded type can generate a strong and accurate force because the device is grounded and not affected by the reaction force. However, there is a problem 
whereby the range of the force to be presented is limited within the cube. Additionally, although this device type can apply a multi-directional force using multiple threads, the operating range of the fingertip is more limited owing to interference amongst the threads. SPIDAR has been under development for a long time and comes in various types [8]. SPIDAR-S [9], which presents a force sensation to the user's finger with a single thread, and SPIDAR-MF [10], which corresponds to wrist twisting using a rotatable frame structure, have been proposed to solve the workspace problem. However, SPIDAR-S does not assume a multiple degree-of-freedom (DoF) force sensation, and SPIDAR-MF focuses on preventing the interference of threads corresponding to multiple fingers.

Based on these considerations, a haptic device using a thread currently has three requirements: suppressing the generation of the force to a position except for the intended position; preventing the interference between the body and the threads; presenting a force sensation within a wide range. The proposed haptic device satisfies these three requirements at the cost of sacrificing the accuracy of force direction. The problem of the repulsive force has been solved by developing the device as a grounded type. The interference between the body and the threads is prevented by using one thread from the fishing rod. Finally, the presentation of the force sensation within a wider range is realized using a method for driving the base of the fishing rod.

\section{Device Configuration}

The proposed device is shown in Fig. 1. A thread coming out of a fishing rod is attached to the user's fingertip end-effector. To wind up the thread, a DC motor (Maxon, 135079, $10 \mathrm{~W}$ ) is attached to the reel and controlled using PWM control. To present the multi-directional force using the fishing rod, two servomotors (JX Servo, C 70 Digital Servo, $92 \mathrm{~W}$ ) are mounted onto the base supporting the fishing rod, and a pan-tilt mechanism is adopted. The fingertip position is detected by an optical motion capturing system (OptiTrack, V 120: Trio) installed around the device, and optical markers (retroreflective balls) are attached to the fingertip end-effector. With the abovementioned device configuration, the winding of the thread attached to the endeffector and the posture of the fishing rod are controlled to present the force sensation.

Three effects are expected when using the fishing rod as a haptic display. First, compared with the case of multi-threads, the calculation of the force is simpler because the tension direction of the thread matches the direction of the force presented to the fingertip. Second, because the fishing rod is lightweight, it is possible to control the posture of the fishing rod at high speed, even when using a motor with relatively low torque. Third, the fishing rod can innately present a strong force to the fingertip. Although the necessary torque is calculated from the length of the fishing rod and thread tension, it can be suppressed when a strong force is required because the bending of the fishing rod has the effect of equivalently shortening the length of the fishing rod such as right figure of Fig. 1. In this case, however, the accuracy of the force direction is reduced. 


\section{Control Method}

In this study, we considered a control method for presenting a directional force. Accordingly, we limited the investigation to the case of a vertical force; that is, the thread attached to the fingertip always exerts a straight upward pull. The optical motion capturing system detects the position of the fingertip and the proposed device vertically pulls the thread attached to the fingertip by controlling the winding of the thread and the posture of the fishing rod.

In this case, the fingertip position becomes the input information of the control system. When introducing this device into a virtual reality application, it is necessary to assess whether it touches the object in the virtual space or not, and to change the presenting magnitude of the force. Additionally, it is important to change the magnitude of the force to express the softness suitable to a virtual object. In other words, the direction and magnitude of the force presented to the fingertip are the targets that should be realized by controlling the device.

In the case of a serial link type haptic device, and particularly with regard to the control of an impedance device type such as PHANToM [4], the position of the end effector is the input, as in the present situation, while the direction and magnitude of the presented force are the targets to be realized. Because a conventional serial link haptic device does not deform the link mechanism itself, it is easy to measure the position of the end effector by carrying out forward kinematics calculations to obtain the torque required by the motor through inverse dynamics calculations. In our case, it is currently difficult to derive a calculation formula that considers the bending of the fishing rod in relation to the force presented at the fingertip using the proposed device.

Thus, we initially attempted to perform feedback control using the difference between the target direction of the force and the current direction of the thread such as Fig. 2(a). The optical marker was not only attached to the fingertip end-effector, but also to the thread (Fig. 2(b)), and the direction of the thread was obtained.
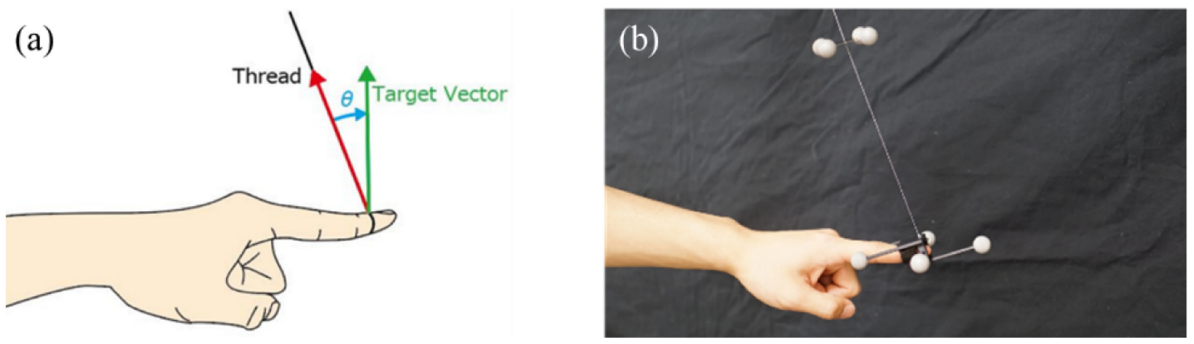

Fig. 2. Overview of feedback control; (a) consideration: (b) actual situation.

However, when the device was actually operated through feedback control, the problem of thread vibration occurred. One of the causes for this is that the sampling rate of the optical tracking was $120 \mathrm{~Hz}$, which is close to the resonant frequency of the thread itself and increased the control difficulty. Additionally, the resonant frequency of the thread dynamically changed with the change of its length and tension. 
Based on the abovementioned trial, we considered that it is currently difficult to control the fishing rod using feedback control. Thus, we decided to control the fishing rod using feedforward control. As mentioned above, the measurement information in the proposed device is the fingertip position, and the target values are the magnitude and direction of the force presented at the fingertip. This time, the target direction of the force was fixed to be vertical. Moreover, it is desirable that the winding force of the thread and the angle of the fishing rod are obtained as output information. To this end, it was necessary to investigate the relationship between the position of the fingertip and the winding force of the thread, the posture of the fishing rod, and the force generated by the winding force of the thread and posture of the fishing rod. In the next section, we present the measurement and fitting of the relationship between the input and output information.

\section{Measurement}

\subsection{Measurement Method}

In the measurement, the relationship between position $x$ of the fingertip and the duty ratio command value $d$ to the motor used for winding the thread, the attitude $\theta$ of the fishing rod, and the force $f$ generated by the winding force of the thread and the attitude of the fishing rod were measured. The measurement environment is shown in Fig. 3. We attached a 1.2-kg load instead of a finger. The position $x$ was the distance from the device to the load. The angle of the fishing rod is defined as $0^{\circ}$ when it is parallel to the ground. To measure the force of the thread pulling the load, the load was placed onto an electronic balance. The weight of the load was recorded by starting the electronic balance before the measurement, and the traction force was calculated from the change of the electronic balance when the load was pulled by the thread.

The load was placed at $22.5 \mathrm{~cm}, 45.0 \mathrm{~cm}, 56.25 \mathrm{~cm}, 67.5 \mathrm{~cm}, 78.75 \mathrm{~cm}$, and $90.0 \mathrm{~cm}$ with the device position as the origin. The duty ratio of the motor winding the thread at each position was changed by $10 \%$, from $10 \%$ to $100 \%$. Then, the angle of the fishing rod was adjusted such that the thread pulled the load exactly in the vertical direction. Additionally, because the motor of the reel started to rotate when the duty ratio was $5 \%$ or more, measurements were also made when the duty ratio was $5 \%$ at

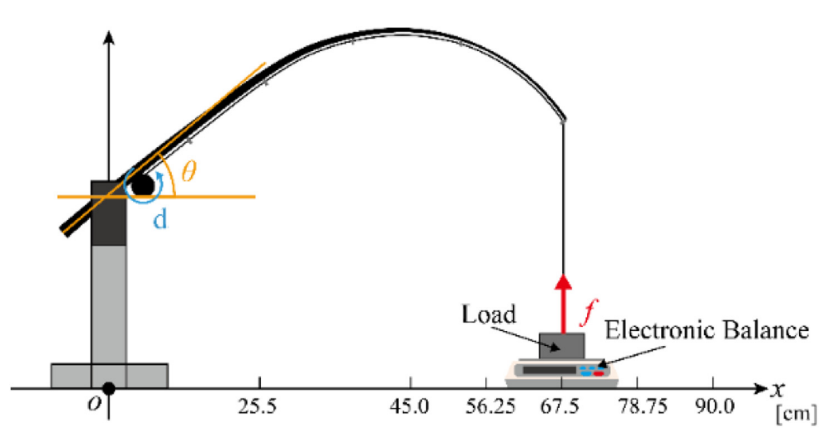

Fig. 3. Measurement environment. 
each position. The measurement of the fishing rod's angle and the traction force to the 11 values of the duty ratio was repeated five times while the position was fixed, and the mean and the standard deviation were calculated.

\subsection{Measurement Result}

The measured means and the standard deviations were plotted in Fig. 4. The relationship amongst location $x$ of the load, duty ratio $d$ of the motor controlling the winding of the thread, and angle $\theta$ in the pitch axis direction of the fishing rod is shown in Fig. 4 (a). Figure 4 (b) shows the relationship amongst location $x$ of the load, the duty ratio $d$ of the reel motor, and the force $f$ of the thread pulling the weight.

In Fig. 4(a) and (b), only one data point existed when the load was set to $90 \mathrm{~cm}$. The total length of the fishing rod used in this study was $90 \mathrm{~cm}$. Additionally, the duty ratio of $5 \%$ was the minimum duty ratio at which the motor of the reel started to rotate (the fishing rod began to bend).

The measurement data obtained when the load was placed at $22.5 \mathrm{~cm}$ and $78.75 \mathrm{~cm}$ was smaller in the number of plots than the data obtained when the load was placed from $45.0 \mathrm{~cm}$ to $67.5 \mathrm{~cm}$. When the load was set to $78.75 \mathrm{~cm}$, the posture of the fishing rod for pulling in the vertical direction was not observed with a duty ratio higher than $40 \%$. Moreover, when the load was installed at $22.5 \mathrm{~cm}$ and the duty ratio exceeded $20 \%$, the fishing rod began to bend not only in the pitch axis direction, but also in the roll axis direction. Therefore, the duty ratio over 30\% was not measured.

According to Fig. 4 (a), the fishing rod's angle increases as the position of the load approaches the device. Similarly, in Fig. 4 (b), the traction force also increases. Increasing the fishing rod's angle is the same as increasing its whip. Therefore, it can be confirmed that the traction force also increases, when the whip of the fishing rod increases.
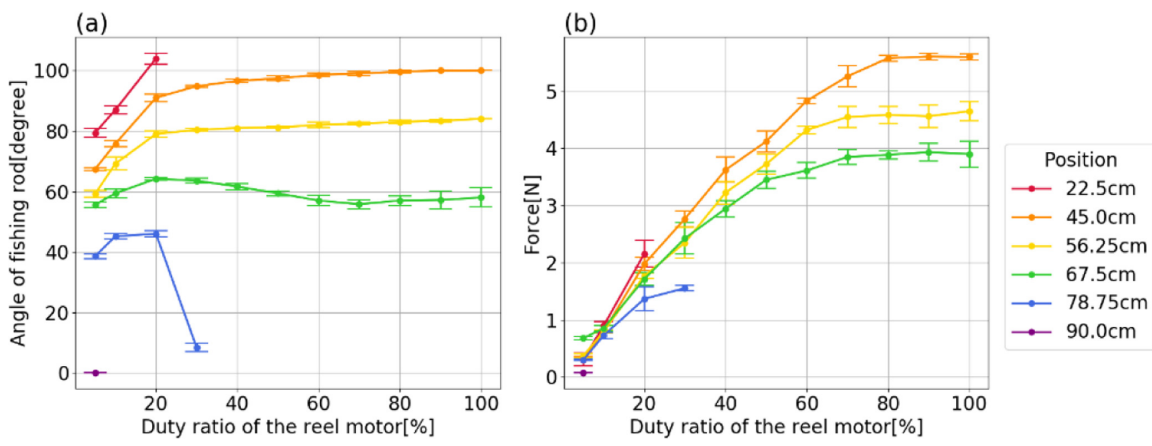

Fig. 4. Measurement results: (a) relationship between duty ratio of motor winding the thread and attitude of fishing rod; (b) relationship between duty ratio of motor winding the thread and pulling force of the thread. 
In the proposed feedforward control, the position $x$ of the fingertip and the magnitude $f$ of the presented force are given, and the duty ratio $d$ required by the motor winding the thread and the angle $\theta$ of the fishing rod must be obtained. From Fig. 4(b), the duty ratio $d$ of the motor winding the thread was derived by inputting the position $x$ of the fingertip and the strength $f$ of the force to be presented. Then, based on Fig. 4 (a), the posture $\theta$ of the fishing rod was determined from the position $x$ of the fingertip and the duty ratio $d$ of the motor.

To realize the abovementioned procedure, the regression equation obtained by the least squares method was derived from the measurement data presented in Fig. 4. The regression curves based on the regression equation are shown for several locations in Fig. 5 and Fig. 6. Figure 5 shows the regression curve obtained from $x$ and $f$ to $d$, using a second order polynomial, while Fig. 6 shows the regression curve obtained from $x$ and $d$ to $\theta$ using a first order polynomial, which indicates that the regression curve adequately represents the data obtained by measurement.

Our control scheme was confirmed by performing actual feedforward control. The amount of force was fixed and the fingertip was moved. As shown in Fig. 7, the thread always pulled the fingertip straight upward, even when the fingertip was moved, which indicates that the desired feedforward control was realized.
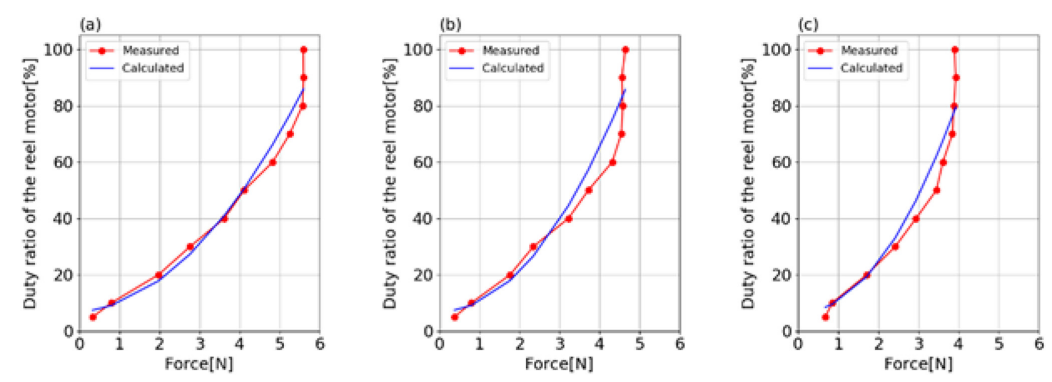

Fig. 5. Comparison of regression curves with measurements of thread pulling force and force winding the thread: (a) $x=45.0 \mathrm{~cm}$; (b) $x=56.25 \mathrm{~cm}$; (c) $x=67.5 \mathrm{~cm}$.
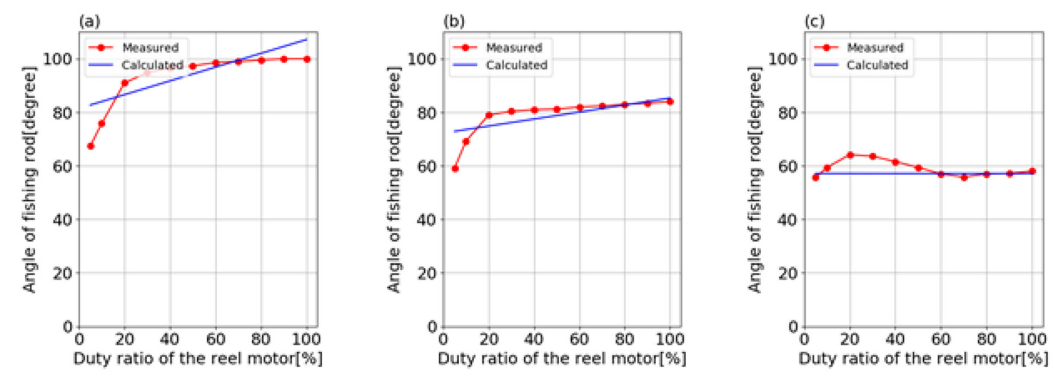

Fig. 6. Comparison of regression curve with measurements for force winding the thread and posture of fishing rod: (a) $x=45.0 \mathrm{~cm}$; (b) $x=56.25 \mathrm{~cm}$; (c) $x=67.5 \mathrm{~cm}$. 

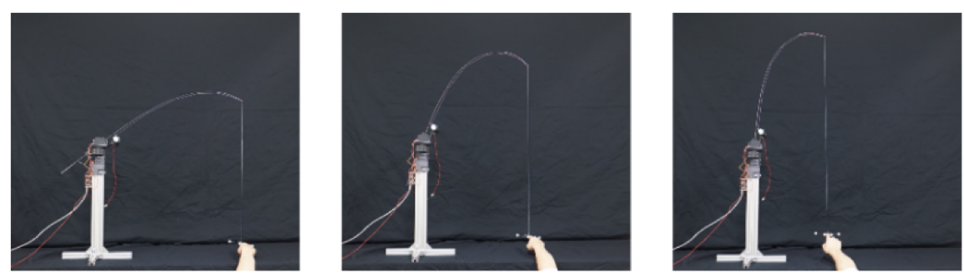

Fig. 7. Validation of control; the fingertip is continuously pulled in vertical direction with the same amount of force.

\section{Conclusion}

This paper proposes a haptic device using a fishing rod and describes the proposed control method that pulls the fingertip in the vertical direction. The relationship between the data was investigated by measuring the position of the fingertip, winding force of the thread, posture of the fishing rod, and force of the thread pulling the fingertip. The regression curves were obtained based on the measured data and incorporated into the control of the device. Thus, it was always possible to pull the fingertip in the vertical direction, even if the fingertip was moved.

However, the proposed control method is obviously a simplification, and must be expanded to all force directions and fingertip positions. The vibration of the fishing rod by quick motion is another issue that must be solved. Additionally, we must clarify the space wherein an accurate force direction can be reproduced, and the space wherein the force direction cannot be reproduced but the force amplitude can be reproduced. This should be coupled with investigations into how much the wrong force direction affects the perception of force sensation and the related task performance.

Furthermore, a user study within a virtual reality environment should be also conducted, both with regard to subjective evaluation, such as softness presentation, and task performance. From now on, we will evaluate the performance of the device with a focus on the user studies.

Acknowledgment. This research was supported by JSPS KAKENHI Grant Number JP18H04110.

\section{References}

1. Aoki, T., Mitake, H., Keoki, D., Hasegawa, S., Sato, M.: Wearable haptic device to present contact sensation based on cutaneous sensation using thin wire. In: ACM International Conference Proceeding Series, pp. 115-122 (2009)

2. Seifi, H., et al.: Haptipedia: accelerating haptic device discovery to support interaction \& engineering design. In: Proceedings of the 2019 CHI Conference on Human Factors in Computing Systems (2019)

3. Barnaby, G., Roudaut, A.: Mantis: A scalable, lightweight and accessible architecture to build multiform force feedback systems. In: UIST 2019-Proceedings of the 32nd Annual ACM Symposium on User Interface Software and Technology, pp. 937-948 (2019) 
4. Jarillo-Silva, A., Domínguez-Ramírez, O.A., Parra-Vega, V., Ordaz-Oliver, J.P.: PHANToM OMNI haptic device: Kinematic and manipulability. In: CERMA 2009-Electronics Robotics and Automotive Mechanics Conference, pp. 193-198. IEEE (2009)

5. Sato, M., Hirata, Y., Kawarada, H.: Space interface device for artificial reality-SPIDAR. Syst. Comput. Jpn. 23, 44-54 (1992)

6. Hirose, M., et al.: HapticGEAR: the development of a wearable force display system for immersive projection displays. In: Proceedings-Virtual Reality Annual International Symposium (2001)

7. Hosseini, M., Meattini, R., Palli, G., Melchiorri, C.: Development of sEMG-driven assistive devices based on twisted string actuation. In: 2017 3rd International Conference on Control, Automation and Robotics (ICCAR), pp. 115-120. IEEE (2017)

8. Sato, M.: Development of string-based force display: SPIDAR. In: 8th International Conference on Virtual Systems and Multimedia (VSMM) (2002)

9. Ma, S., Toshima, M., Honda, K., Akahane, K., Sato, M.: SPIDAR-S: a haptic interface for mobile devices. In: Chen, Y., Christie, M., Tan, W. (eds.) SG 2015. LNCS, vol. 9317, pp. 203-206. Springer, Cham (2017). https://doi.org/10.1007/978-3-319-53838-9_18

10. Liu, L., Miyake, S., Akahane, K., Sato, M.: Development of string-based multi-finger haptic interface SPIDAR-MF. In: Proceedings of 23rd International Conference on Artificial Reality and Telexistence (ICAT), pp. 67-71 IEEE (2013)

Open Access This chapter is licensed under the terms of the Creative Commons Attribution 4.0 International License (http://creativecommons.org/licenses/by/4.0/), which permits use, sharing, adaptation, distribution and reproduction in any medium or format, as long as you give appropriate credit to the original author(s) and the source, provide a link to the Creative Commons license and indicate if changes were made.

The images or other third party material in this chapter are included in the chapter's Creative Commons license, unless indicated otherwise in a credit line to the material. If material is not included in the chapter's Creative Commons license and your intended use is not permitted by statutory regulation or exceeds the permitted use, you will need to obtain permission directly from the copyright holder. 